\title{
Integrability analysis of the Emden-Fowler equation
}

$K S$ GOVINDER $R^{\dagger}$ and $P$ G L LEACH ${ }^{\ddagger 1}$

† Astrophysics and Cosmology Research Unit, School of Mathematical Sciences,

University of KwaZulu-Natal, Durban 4041, Republic of South Africa

Email: govinder@ukzn.ac.za

$\ddagger$ Research Group in Mathematical Physics, Department of Information and

Communication Systems Engineering, School of Sciences, University of the Aegean,

Karlovassi 83 200, Greece

Email: leach@ukzn.ac.za; leach@math.aegean.gr

Received January 6, 2007; Accepted in Revised Form April 6, 2007

\begin{abstract}
The Emden-Fowler equation of index $n$ is studied utilising the techniques of Lie and Painlevé analysis. For general $n$ information about the integrability of this equation is obtained. The link between these two types of analyses is explored. The special cases of $n=-3,2$ are also examined. As a result of the Painlevé analysis new second-order equations possessing the Painlevé property are found.
\end{abstract}

\section{Introduction}

The Emden-Fowler equation [41, 15, 20, 21, 22, 23] has attracted much attention over the years. Wong, in his review of 1976 [61], contains over 100 references, but even these were selective. Subsequently a plethora of papers has appeared devoted to a study of this ubiquitous equation. The most general form studied today ${ }^{2}$ is

$$
Y^{\prime \prime}+p(X) Y^{\prime}+q(X) Y=r(X) Y^{n} .
$$

However, it is well-known [52] that a Kummer-Liouville transformation [40, 48] converts (1.1) into standard form, videlicet

$$
y^{\prime \prime}=f(x) y^{n} .
$$

It is this form of the equation to which we confine our analysis. Equation (1.2) has become increasingly important as it arises in the modelling of many physical systems.

\section{Copyright (c) 2007 by KS Govinder and PGL Leach}

\footnotetext{
${ }^{1}$ Permanent address: School of Mathematical Sciences, University of KwaZulu-Natal, Durban 4041, Republic of South Africa

${ }^{2}$ We observe that Chandrasekar et al [11] would even include $\ddot{x}+\alpha x \dot{x}+\beta x^{3}=0$ within the pantheon of equations of Emden type, but this does seem to be stretching the word 'modified' a little beyond its standard meaning.
} 
It is perhaps best known for its occurrence as the quintessential equation in the study of the shear-free spherically symmetric perfect fluid motion in Cosmology when $n=2$ $[59,51,32,28]$.

We study (1.2) from the viewpoints of Lie symmetries and the Painlevé analysis. In general (1.2) does not possess any Lie point symmetries nor can one easily say anything about its possession of the Painlevé property. However, for an appropriate $f(x)(1.2)$ does possess at least one Lie point symmetry. We analyse (1.2) for these instances and also consider the conditions for it to possess more than one Lie point symmetry, thereby enabling the reduction to quadratures. (See also in this respect $[7,8]$.) In addition we show under what conditions (1.2) (with only one Lie point symmetry) can be reduced to quadratures.

We also undertake a Painlevé analysis of (1.2) (suitably transformed) and discuss its possession of the full Painlevé property. We comment on a possible link between possession of the Painlevé property and explicit integration of the equation. Our interest is in the relationship between the equation possessing the Painleve property and the evaluation of the quadrature to which it is reduced via the Lie analysis. We also consider the special cases of $n=-3,2$ and show how these values affect the analysis.

We noted above that the Emden-Fowler equation has attracted much attention over the years. A paper of some particular relevance is that by Euler [17] since there symmetry and singularity analyses are used. In Section 5 we provide a comparison of the two works.

\section{Lie Analysis}

An $n$ th-order differential equation

$$
E\left(x, y, y^{\prime}, \ldots, y^{(n)}\right)=0
$$

is said to possess the Lie point symmetry

$$
G=\xi(x, y) \partial_{x}+\eta(x, y) \partial_{y}
$$

if

$$
G^{[n]} E_{\left.\right|_{E=0}}=0,
$$

where $[50]$

$$
G^{[n]}=G+\sum_{j=1}^{n}\left(\eta^{(j)}-\sum_{k=0}^{j-1}\left(\begin{array}{l}
j \\
k
\end{array}\right) y^{(k+1)} \xi^{(j-k)}\right) \partial_{y^{(j)}}
$$

is the $n$th extension of $G$ needed to contend with the $n$th derivatives of $y$ in (2.1). Note that we are restricting our analysis to scalar ordinary differential equations. The concept 
of Lie point symmetries applies equally to systems of equations and to partial differential equations. In those instances the $\xi, \eta, x$ and $y$ take suitable indices. (See eg [9, 54].) The action of $G^{[n]}$ on (2.1), ie (2.3), gives a system of linear partial differential equations which is solved to obtain $G$. Thereafter $G$ can be used either to transform (2.1) appropriately (usually into autonomous form) or reduce the order. The aim, of course, is to reduce the $n$ th-order equation to zeroth order.

In the case of the Emden-Fowler equation

$$
y^{\prime \prime}=f(x) y^{n}
$$

it is easily verified that $\xi$ and $\eta$ in (2.2) must have the form

$$
\xi=a(x) \quad \eta=c(x) y+d(x) .
$$

We therefore begin the Lie analysis of (2.5) by assuming the form

$$
G=a(x) \partial_{x}+(c(x) y+d(x)) \partial_{y}
$$

for a symmetry of (2.5). The action of $G^{[2]}$ on (2.5) results in the system

$$
\begin{aligned}
-2 f a^{\prime}+c f & =a f^{\prime}+n c f \\
n f d & =0 \\
c^{\prime \prime} & =0 \\
d^{\prime \prime} & =0 \\
2 c^{\prime}-a^{\prime \prime} & =0 .
\end{aligned}
$$

(These are obtained by equating coefficients of different powers of $y^{\prime}$ and $y$ to zero.) We immediately integrate (2.12) to obtain

$$
c=\frac{1}{2}\left(a^{\prime}+k\right),
$$

where $k$ is the constant of integration, and observe that $d$ in (2.9) is zero. Thus (2.11) is identically satisfied. Note that (2.9) and (2.10) coalesce in the case $n=2$ and $c$ and $f$ are related via $d$ (See (4.8).). Note also that (2.8) can be rewritten as

$$
a f^{\prime}+\left(\frac{n-1}{2}\left(a^{\prime}+k\right)+2 a^{\prime}\right) f=0
$$

which is special in the case $n=-3$ and $k=0$. We return to these cases later. The cases $n=0,1$ are equivalent as the equation is then linear. Linear second-order differential equations have eight Lie point symmetries which form the Lie algebra $s \ell(3, R)$. (See [30] for a recent proof and references therein.)

For general $n$ we write (2.14) as

$$
\frac{f^{\prime}}{f}=-\left(\frac{n+3}{2}\right) \frac{a^{\prime}}{a}-\left(\frac{n-1}{2}\right) \frac{k}{a}
$$


from which

$$
f=K a^{-(n+3) / 2} \exp \left[-\frac{(n-1) k}{2} \int \frac{\mathrm{d} x}{a}\right] .
$$

The differential equation for $a$ is (from (2.10) and (2.13))

$$
a^{\prime \prime \prime}=0,
$$

whence

$$
a=A_{0}+A_{1} x+A_{2} x^{2} .
$$

Equation (2.5) has the symmetry

$$
G_{1}=a \partial_{x}+\frac{1}{2}\left(a^{\prime}+k\right) y \partial_{y}
$$

if $f(x)$ is given by (2.16).

Using the transformation

$$
\begin{aligned}
X & =\int \frac{\mathrm{d} x}{a} \\
Y & =y a^{\frac{1}{2}} \exp \left[-k \int \frac{\mathrm{d} x}{2 a}\right]
\end{aligned}
$$

we rewrite (2.5) in autonomous form, videlicet

$$
Y^{\prime \prime}+k Y^{\prime}+\left(\Delta+\frac{k^{2}}{4}\right) Y=K Y^{n},
$$

where

$$
\Delta=A_{0} A_{2}-\frac{1}{4} A_{1}^{2} .
$$

Reduction via

$$
u=Y \quad v=Y^{\prime}
$$

results in an Abel's equation of the second kind, videlicet

$$
v v^{\prime}=K u^{n}-k v-\left(\Delta+\frac{k^{2}}{4}\right) u,
$$

the solution of which (though it exists in principle) is unobvious. Thus (2.5) can be reduced to a first-order equation provided $f$ is given by (2.16) and $a$ by (2.18).

To reduce (2.22) to quadratures we require that the equation which arises after the first reduction of order possess at least one Lie point symmetry. (The fact that this reduced equation is of first order and so possesses an infinite number of Lie point symmetries is moot. The determination of these symmetries remains an intractable problem [9].) 
If (2.22) possesses two Lie point symmetries, $G_{1}$ and $G_{2}$ say, and $\left[G_{1}, G_{2}\right]=\lambda G_{1}(\lambda$ an arbitrary constant usually 1 or 0$)$, the reduction via $G_{1}$ results in a first-order equation with $G_{2}$ (suitably extended) as a point symmetry [54]. We therefore further examine (2.22) to determine under which circumstances it possesses two point symmetries.

\section{Setting}

$$
G=\tilde{a}(X) \partial_{X}+\tilde{c}(X) Y \partial_{Y}
$$

where we have removed $\tilde{d}(X)$ in the coefficient of $\partial_{Y}$ since the form of (2.22) implies $\tilde{d}(X)=0$, we require

$$
G^{[2]} N_{\left.\right|_{N=0}}=0,
$$

where we have rewritten $(2.22)$ as $N\left(Y, Y^{\prime}, Y^{\prime \prime}\right)=0$. The operation (2.27) results in the system

$$
\begin{aligned}
\tilde{c}-2 \tilde{a}^{\prime} & =n \tilde{c} \\
2 \tilde{c}^{\prime}+k \tilde{a}^{\prime}-\tilde{a}^{\prime \prime} & =0 \\
\tilde{c}^{\prime \prime}+2 M \tilde{a}^{\prime}+k \tilde{c}^{\prime} & =\tilde{c},
\end{aligned}
$$

where

$$
M=\Delta+\frac{k^{2}}{4} .
$$

The function $\tilde{c}$ is determined from (2.28), videlicet

$$
\tilde{c}=-\frac{2 \tilde{a}^{\prime}}{n-1} .
$$

The differential equation for $\tilde{a}$ now becomes (via (2.29) and (2.32))

$$
\frac{n+3}{n-1} \tilde{a}^{\prime \prime}-k \tilde{a}^{\prime}=0
$$

and so $\tilde{a}$ is given by

$$
\tilde{a}=\tilde{A}_{0}+\tilde{A}_{1} \exp \left(\left(\frac{n-1}{n+3}\right) k X\right) .
$$

Equation (2.30) now becomes the consistency condition

$$
\frac{\tilde{a}^{\prime \prime \prime}}{n-1}-M \tilde{a}^{\prime}+\frac{k \tilde{a}^{\prime \prime}}{n-1}=0 .
$$

If we invoke $(2.33),(2.35)$ is only satisfied if

$$
M=\frac{2 k^{2}(n+1)}{(n+3)^{2}}
$$


from which (via (2.31))

$$
\Delta=-\left[\frac{k(n-1)}{2(n+3)}\right]^{2} .
$$

This implies

$$
\frac{1}{4} A_{1}^{2}-A_{0} A_{2}=\left[\frac{k(n-1)}{2(n+3)}\right]^{2}
$$

which further implies that the equation for a, videlicet (2.18), has real roots!

From (2.26) and (2.34), (2.22) has the two Lie point symmetries

$$
\begin{aligned}
G_{1} & =\partial_{X} \\
G_{2} & =\exp \left[\left(\frac{n-1}{n+3}\right] k X\right)\left(\partial_{X}-\frac{2 k Y}{n+3} \partial_{Y}\right)
\end{aligned}
$$

provided (2.38) holds.

Under the transformation

$$
\mathcal{X}=\frac{\exp \left[\left(\frac{n-1}{n+3}\right) k X\right]}{\frac{(n-1)}{(n+3)} k} \quad \mathcal{Y}=Y \exp \left[\left(\frac{2 k}{n+3}\right) X\right]
$$

(2.22) becomes

$$
\mathcal{Y}^{\prime \prime}=K \mathcal{Y}^{n},
$$

(2.39)-(2.40) are transformed to

$$
\begin{aligned}
& X_{1}=(1-n) \mathcal{X} \partial \mathcal{X}+2 \mathcal{Y} \partial \mathcal{Y} \\
& X_{2}=\partial \mathcal{X}
\end{aligned}
$$

and

$$
\left[X_{1}, X_{2}\right]=(1-n) X_{2} \text {. }
$$

We can now evaluate $f$ as

$$
f=K A_{2}^{-(n+3) / 2}\left(x+\frac{A_{1}}{2 A_{2}}-\frac{k}{2 A_{2}} \frac{n-1}{n+3}\right)^{-(n+3)} .
$$

Equation (2.42) can be reduced using $X_{2}$ and then $X_{1}$ to the quadrature

$$
\mathcal{X}-\mathcal{X}_{0}= \pm \int \frac{\mathrm{d} \mathcal{Y}}{\left(\frac{K}{n+1} \mathcal{Y}^{n+1}+K_{1}\right)^{\frac{1}{2}}}
$$


where $\mathcal{X}_{0}$ and $K_{1}$ are arbitrary constants of integration.

Remark: We observe that (2.22) can also be reduced to quadratures in the case $k=0$. This suggests that a group theoretic approach [47] is not applicable in this case. However, an extension of the Lie theory to nonlocal symmetries [29] reveals that

$$
y^{\prime \prime}+M y=K y^{n}
$$

possesses an additional (nonlocal) symmetry. The function $f$ now becomes

$$
f=K\left(A_{0}+A_{1} x+A_{2} x 2\right)^{-(n+3) / 2} .
$$

(See also [37] for a treatment of the two-symmetries case for $f=x^{m}$.)

Thus for (2.5) to be reduced to quadratures $f$ must be given by (2.16), $a$ by (2.18) and (2.38) must hold.

\section{Painlevé Analysis}

From $\S 2$ it is evident that the Lie theory of differential equations (and its extensions) is rather exhaustive in its treatment of the Emden-Fowler equation. We now investigate its possession of the Painlevé property to determine whether any further interesting information can be obtained. (See the excellent report [14] for a lucid introduction to this technique of analysis.) An ordinary differential equation is said to possess the Painlevé property if its general solution has no critical points [14]. When one considers the evidence encountered in the literature, it is conjectured that an equation possessing the Painlevé property is integrable in the sense that it possesses a solution which is analytic away from isolated movable polelike singularities ${ }^{3}$. The method of analysis we employ is not due to Painlevé [56], but was introduced in 1889 by Kowalevski [38, 39]. It is the method of polelike expansions that has recently been popularised by Ablowitz et al [1].

Before we proceed with the analysis some comments are in order. It is well-known that Painlevé worked on the classification of second-order ordinary differential equations of the first degree to determine those which possess the Painlevé property (though he presumably did not term it so). The work, completed by Gambier [24], constitutes a complete classification of all first-degree second-order differential equations that are rational in the both the dependent variable and its first derivative and analytic in the independent variable. However, we note that (2.42) does not naturally fall into the classes of equations listed in $[24,35]$ as i) $n$ can be rational and ii) these lists are complete up to a homographic transformation. Thus a Painlevé analysis of (2.42) should highlight interesting properties of this equation.

\footnotetext{
${ }^{3}$ There is also the weaker concept of integrability over a sector of the complex plane, ie the solution is an analytic function away from an isolated movable branch-point singularity in a region defined by the branch point and its cuts.
} 
Having motivated the need for the Painlevé analysis of (2.42) we study the equation in the form

$$
y^{\prime \prime}=y^{n},
$$

where the $K$ in (2.42) has been removed through the rescaling of $y$. The analysis essentially involves assuming a Laurent series expansion for the dependent variable about some point $x-x_{0}$. The procedure has three accepted parts ${ }^{4}$. The first is the determination of the leading-order behaviour by the substitution of

$$
y=\alpha \chi^{p}
$$

where $\alpha$ and $p$ are constants to be determined and

$$
\chi=x-x_{0},
$$

where $x_{0}$ is the location of the putative movable pole, into the equation of interest. After the calculation of $p$ and $\alpha$ the expression

$$
y=\alpha \chi^{p}+\beta \chi^{i+p}
$$

is substituted into the dominant terms of the equation to determine the indices $i$ (at which the remaining constants of integration arise) by requiring that the coefficient of terms linear in $\beta$ is zero. Finally the truncated Laurent expansion

$$
y=\alpha \chi^{p}+\delta_{1} \chi^{p+1}+\cdots+\delta_{i+p} \chi^{i+p}
$$

is substituted into the original equation to verify that no incompatibilities occur that violate the arbitrariness of the constants of integration which arise at the indices.

The substitution of (3.2) into (3.1) yields

$$
p=-\frac{2}{n-1} \quad \alpha^{n-1}=\frac{2(n+1)}{(n-1)^{2}}
$$

with both terms in (3.1) being obviously dominant, and (3.4) into (3.1) yields

$$
i=-1, \frac{2(n+1)}{n-1} \text {. }
$$

The -1 is to be expected [14] and the second constant of integration (in addition to $x_{0}$ ) arises as the coefficient of $\chi^{2(n+1) /(n-1)}$ in the Laurent expansion for $y$. Note that for the implementation of the method $p$ in (3.6) must be a negative integer. This arises for the values $n=2,3$. The nongeneric index arises at $i=6,4$ respectively. We do not need to substitute the truncated Laurent expansion into (3.1) to check for incompatibilities at the index as all the terms in (3.1) are dominant and this suffices for an equation of the second order [19]. We note that (2.47) can be easily evaluated for these values of $n$.

\footnotetext{
${ }^{4}$ For a broader interpretation of the analysis see Géronimo et al [25].
} 
For $n \neq 2,3 p$ and $i$ are rational and the possibility that the solution of (3.1) possesses algebraic branch points exists. However, we can transform the denominator of $p$ away by setting either

$$
Y=y^{n-1} \quad X=x
$$

or

$$
Y=y \quad X=x^{1 /(n-1)} .
$$

The transformation (3.9) is homographic (and thereby preserves the Painlevé property $[14,24])$. Consequently there is no need to analyse further the effect of this transformation.

While (3.8) is not homographic, it does preserve the polynomial form of (3.1) (for integer $n$ ) and so is an acceptable transformation [14]. The equation becomes

$$
Y^{\prime \prime}=(n-1) Y^{2}+\frac{n-2}{n-1} \frac{Y^{\prime 2}}{Y} .
$$

Two cases arise:

i) $p=-2, i=-1,2(n+1) /(n-1), \alpha=\frac{2(n+1)}{(n-1)^{2}}$

ii) $p=n-1, i=-1,0, \alpha-$ arbitrary.

Case ii) arises in the instance that the two derivative terms in (3.10) are dominant only. However, this is equivalent to only the first term in (3.1) being dominant and so is discussed no further in terms of (3.1). In case i) $i$ is a positive integer only when $n=-3,-1,2,3,5$ (with the corresponding $i$ values $1,0,6,4,3$ respectively). The case $n=-1$ can be immediately discounted as $i=0$ implies that $\alpha$ is arbitrary. However, we note that $\alpha$, in case i), is fixed (and is in fact zero!). This points to the introduction of logarithmic terms in the expansion for $Y$ which violates the integrability of (3.10) in terms of analytic functions [58].

In terms of (3.10) as an equation in its own right it is of interest to examine the family $p=n-1$. For $n=2,3,5, p>0$. Ordinarily this would suggest the transformation

$$
\mathcal{Y}=\frac{1}{Y}
$$

to make $p$ negative. However, as we have specific values for $n$ we can resort to looking up the appropriate equations in $[24,35]$. We find that the equations corresponding to $n=-3,2,3,5$ are equations $(22),(2),(18)$ and (21) of [24] respectively. Thus (3.10) has the Painlevé property for $n=-3,2,3,5$.

Note that, as $n$ is a physical constant related to the ratio of specific heats in the astrophysical context [42], it can be rational. In the subsequent analysis we expressly ignore integer $n$. A study of the relationship (3.6) reveals that $p$ is a negative integer for $1<n<5 / 3$. For $p=-2 /(n-1) \in \mathbb{Z}<0, i=2(n+1) /(n-1)=2-2 p \in \mathbb{Z}>0$ (in 
(3.7)). This points to (3.1) possessing the full Painlevé property. In this instance we do not have recourse to the lists in $[24,35]$ as these are concerned with rational functions of the dependent variable. It should be noted that no such restriction was originally intended by Painlevé $[56,55]$. We do not have to substitute the truncated Laurent expansion (3.5) into (3.1) to verify that no incompatibilities arise at the index as both terms are dominant. Thus we introduce equations of the form

$$
y^{\prime \prime}=y^{(p+2) / p}, \quad p \in \mathbb{N}, p>2,
$$

equally

$$
y^{\prime \prime p}=y^{p+2}, \quad p \in \mathbb{N}, p>2,
$$

into the literature as part of the class of second-order ordinary differential equations possessing the Painlevé property.

\section{The special cases $n=-3,2$}

We have seen above that in the case $n=-3,2,(1.2)$ can be reduced to a quadrature that can be evaluated. However, these cases have a deeper significance that the Lie analysis in $\S 2$ did not reveal.

For $n=-3$ and $k=0$ we solve (2.14) to obtain

$$
f=\tilde{K},
$$

where $\tilde{K}$ is an arbitrary constant. The solution for $a$ (obtained from (2.10) and (2.13)) is

$$
a=A_{0}+A_{1} x+A_{2} x^{2}
$$

with $c$ given by

$$
c=\frac{A_{1}}{2}+A_{2} x
$$

and $d=0$ as before. Equation (1.2) now has the form

$$
y^{\prime \prime}=\tilde{K} y^{-3},
$$

which is the well-known Ermakov-Pinney equation [16, 57], and has the three Lie point symmetries

$$
\begin{aligned}
G_{1} & =\partial_{x} \\
G_{2} & =2 x \partial_{x}+y \partial_{y} \\
G_{3} & =x 2 \partial_{x}+x y \partial_{y}
\end{aligned}
$$

which form the Lie algebra $s \ell(2, R)$. (See also $[43,36,46]$.) This Lie algebra is not solvable, but as we are only concerned with a second-order equation, it is sufficient to reduce (4.4) to quadratures. 
For $n=2,(2.9)$ and (2.10) coalesce into

$$
2 f d-c^{\prime \prime}=0 .
$$

The equation (2.11) causes our analysis to separate into two cases. In the case $d=0$ the analysis for general $n$ applies. We observe that the functions $f$ for which (1.2) can be integrated fall into the class given by Srivastava [59].

The case of $d \neq 0$ has two further subcases. The first is of constant $d$, ie $d=D_{0}$. In this case $f$ is given by

$$
f=\frac{c^{\prime \prime}}{2 d},
$$

with

$$
c=\frac{1}{2} a^{\prime}-\gamma
$$

from (2.12). To find the explicit form for $f$ in (4.9) we need the function $a$ which is obtained by solving

$$
2 a a^{i v}+5 a^{\prime} a^{\prime \prime \prime}-2 \gamma a^{\prime \prime \prime}=0 .
$$

For $\gamma=0$ (4.11) has three symmetries and can be formally integrated ${ }^{5}$. When $\gamma \neq 0$, there are only two symmetries and (4.11) can only be partially integrated.

In the case $d=D_{0}+D_{1} x, f$ has the same form as in (4.9). The equivalent of (4.11) is now

$$
2 a a^{i v} d+5 a^{\prime} a^{\prime \prime \prime} d-2 a a^{\prime \prime \prime} d^{\prime}-2 \gamma a^{\prime \prime \prime} d=0 .
$$

Using the transformation

$$
\eta=\frac{D_{1}}{D_{0}+D_{1} x} \quad \zeta=\frac{a D_{1}^{2}}{\left(D_{0}+D_{1} x\right)^{2}}
$$

we rewrite (4.12) as (with primes denoting differentiation with respect to $\eta$ )

$$
2 \zeta \zeta^{i v}+5 \zeta^{\prime} \zeta^{\prime \prime \prime}+2 \gamma \zeta^{\prime \prime \prime}=0
$$

which has the same form as (4.11). Thus the cases of $d=D_{0}$ and $d=D_{0}+D_{1} x$ reduce to the analysis of the single equation (4.11).

Given a solution to (4.11) we can find $f$ using (4.9) and subsequently transform (1.2) to autonomous form. The resulting equations are of the form (2.22) and the analysis that follows (2.22) applies.

\footnotetext{
${ }^{5}$ The integration is eased by the existence of a nonlocal symmetry which becomes local on reduction of order by one [44]. Equation (4.11) with $\gamma=0$ has attracted attention because of its interesting properties $[18,53]$.
} 
However, we still need to solve the equation for $a$. We firstly consider eq (4.11) with $\gamma=0$ :

$$
2 a a^{i v}+5 a^{\prime} a^{\prime \prime \prime}=0
$$

In spite of the fact that (4.15) only has the three symmetries [33]

$$
G_{1}=\partial_{x}, \quad G_{2}=x \partial_{x} \quad \text { and } \quad G_{3}=a \frac{\partial}{\partial a}
$$

we can reduce it to quadratures via a symmetry reduction. If we reduce (4.15) using $G_{1}$, $G_{2}$ and $G_{3}$ in turn, we obtain an Abel's equation of the second kind, the solution of which is unobvious. The proper route to the reduction is via hidden symmetries $[2,3]$.

The reduction of (4.15) via $G_{1}$ results in

$$
2 u\left(v^{2} v^{\prime \prime \prime}+4 v v^{\prime} v^{\prime \prime}+v^{\prime 3}\right)+5\left(v^{2} v^{\prime \prime}+v v^{\prime 2}\right)=0,
$$

where

$$
u=a, \quad v=a^{\prime} .
$$

A Lie analysis of (4.17) produces three instead of the expected two Lie point symmetries, videlicet

$$
\begin{aligned}
G_{1}^{\prime} & =u \frac{\partial}{\partial u} \\
G_{2}^{\prime} & =v \frac{\partial}{\partial v} \\
G_{3}^{\prime} & =2 u^{2} \frac{\partial}{\partial u}+u v \frac{\partial}{\partial u} .
\end{aligned}
$$

The 'new' point symmetry $G_{3}^{\prime}$ is not a descendant of any of the point symmetries in (4.16), but comes from the nonlocal symmetry [4]

$$
G_{4}=3\left(\int a \mathrm{~d} x\right) \partial_{x}+2 a^{2} \partial_{a}
$$

and is hence a Type II hidden symmetry [2]. This hidden symmetry is the appropriate one for further reduction of (4.17). Using

$$
t=v u^{-1 / 2} \quad w=\frac{1}{2}\left(v^{\prime} u^{3 / 2}-\frac{1}{2} v u^{-1 / 2}\right)^{2}
$$

we obtain

$$
w^{\prime \prime}+3 w^{\prime}+2 w=0
$$

which is trivially solved. Reversing the transformations we obtain

$$
x-x_{0}=\int \frac{\mathrm{d} u}{(-K u 3 / 6-L u 2 / 2-2 P u-2 Q)^{3 / 2}},
$$


where $K, L, P$ and $Q$ are constants of integration and

$$
u=\int \frac{1}{a^{3 / 2}} .
$$

It is interesting to note that the trivial cases of setting all except one (in turn) of $K, L, P$ and $Q$ to zero produce functions $f$ that are subclasses of that of Srivastava [59].

When $\gamma \neq 0$, equation (4.11) has only the two point symmetries

$$
G_{1}=\partial_{x} \quad \text { and } \quad G_{2}=x \partial_{x}+a \partial_{a}
$$

Unfortunately reduction using $G_{1}$ does not produce any hidden symmetries. However, it is of interest to test (4.11) for integrability using the Painlevé analysis. In spite of transforming (4.11) to a suitable form for the analysis, we find that one of the indices occurs at 0 and the other at $-1\left(1+2 a_{p}\right) / 2$, where $a_{p}$ is the coefficient of the pole. To pass the Painlevé test $i$ must be (at least) rational. This fixes $a_{p}$ which contradicts the implication of $i=0$ that $a_{p}$ is arbitrary. The analysis can only be continued by introducing logarithmic terms into the expansion. Thus (4.11) does not possess the full Painlevé property. It has been observed [27] that some information about the partial solution of (4.11) can be obtained from considering the different families of expansions for $y$. This information is naturally contained in (4.23).

\section{Discussion}

The Emden-Fowler equation

$$
y^{\prime \prime}=f(x) y^{n}
$$

has been shown to be integrable (for certain functions $f(x)$ ) for all $n$ (including rational values) by considering a Lie analysis. It was further shown that, if (5.1) possesses two Lie symmetries, it can always be transformed to

$$
Y^{\prime \prime}=Y^{n} \text {. }
$$

(Of course (5.1) can be reduced to quadratures if it has two point symmetries. We mention the transformation to (5.2) so that comparison can be made with the Painlevé property.) This point must be emphasised - all Emden-Fowler equations with two symmetries (which form the Lie algebra $A_{2}$ ) can be transformed to (5.2) and the solution to the original equation is obtained from the solution of (5.2) via the same transformation.

The Painlevé analysis of (5.2) reveals integrability only for restricted values of $n$. It is remarkable that, only for these restricted integer values, the quadrature (2.47) can be evaluated. This does reinforce the close relationship between the Lie and Painlevé analyses observed previously $[28,45,49,5,6]$. In the case of rational $n$, it was shown that, for specific values of $n$ in the range $\left(1, \frac{5}{3}\right],(5.2)$ possesses the Painlevé property. While the 
quadrature (2.47) cannot, as yet, be evaluated for these values, noting the results in [60] and [10] we believe that the evaluation thereof is only a matter of time and effort.

In the Introduction we stated that we would compare the present paper with one of Euler [17] which commences with an equation of the same form as our $(1.1)^{6}$. One notable difference is the discussion by Euler of the transformation of (1.1) in the case of $n=3$ into a special case of the second of the equations for the Painleve transcendents. Since the transformation is point and the Painlevé six are notorious for the general absence of Lie point symmetries, there is no question of an overlap between the material of the two papers in this respect since we are concerned with the existence of at least one Lie point symmetry.

In our analysis we immediately reduced the general form of (1.1) to the normal form, (1.2), by means of a standard point transformation. Euler keeps the general form. For us the essential properties of (1.1) are encapsulated in (1.2). For Euler it is important to maintain a direct link with the source equation. The difference in approach is a matter of philosophy. Euler does not want an additional, possibly difficult to evaluate, transformation to intervene whereas we are concerned with clarity. We believe that both philosophies have their proper places in the literature.

Another point of departure between the two papers is that Euler devotes a considerable proportion of his paper to the question of the linearisability of (1.1) by means of a nonpoint transformation since it cannot be linearised by means of a point transformation (See also [26] in this regard.).

It should not come as a surprise that the analysis of the Lie point symmetries of Euler's (1.1) and (1.2) display similarities in results. Naturally his conditions are more complex since he has kept the original three functions of time and we have reduced the discussion to just the one essential function, the coefficient of $y^{n}$ in (1.2). One must note that the keeping of the three functions does lead to a greater complexity of expression for the conditions on the functions and thus there is a concomitant increase in the possibility of error. For example [17, p 327] the solution given for $f_{3}(t)$ at the bottom of the page is manifestly wrong. The condition imposed on $f_{3}$ is simply a transformation of a variant of the Painlevé-Ince equation. Generally the equations for $f_{3}$ look more complicated and those which are given without ellipses may be interesting to study in their own rights.

What is not addressed by Euler [17] is the singularity analysis which is at the core of the present paper. In particular we have extended the class of Emden-Fowler equations for which the singularity analysis does present new results and we have related these with the ease of the performance of the quadrature to determine the solution of (1.2) in cases that it possesses two Lie point symmetries.

The method used to implement the Painlevé analysis in this paper was that of 'polelike' expansions. While it is a convenient mechanism, it does contain certain pitfalls into which

\footnotetext{
${ }^{6}$ There are differences in notation rather than form in that Euler uses $f_{1}, f_{2}$ and $f_{3}$ for our $p, q$ and $r$.
} 
the unwary practitioner may fall. We caution here against the requirement of negative $p$ for the order of the pole. This requirement is frequently the first step in the analysis and suggests an immediate transformation of the equation under consideration when $p$ is positive. While the resulting equation may possess the Painlevé property, it must be noted that it could well fall outside the class of equations listed in $[24,35]$. The reason is simple: these lists of equations do not insist on negative $p$ ! An example is the case $n=-3$ considered in $§ 3$. Equation (3.10) possesses the full Painlevé property, but does not occur in $[24,35]$ in its present form. Invoking (3.11) results in an equation that does arise in $[24,35]$ with $p$ now being positive. Thus due caution must be observed in the implementation of the algorithm and the analysis of the results (See also [34] in this respect.). It is little wonder that Painlevé did not see the need for 'le procédé connu de Madame Kowaleski' [55].

We finally note that the Painlevé analysis was restricted to equations of the form (5.2) by requiring that (5.1) possess two Lie point symmetries. However, noting that some of the equations in $[24,35]$ do not possess at least two Lie point symmetries, the investigation of the equation in the form (5.1) would be of some interest. Work on this already been started [13].

\section{Acknowledgements}

It is a pleasure to thank Dr Robert Conte for interesting discussions and clarifications. We also thank the National Research Foundation of South Africa and the Universty of KwaZulu-Natal for their continuing support. PGLL thanks the Department of Information and Communication Systems Engineering of the University of the Aegean, Karlovassi, Greece, for the provision of facilities during the preparation of this work.

\section{References}

[1] Ablowitz MJ, Ramani A \& Segur H, Nonlinear evolution equations and ordinary differential equations of Painlevé type, Lettere al Nuovo Cimento, 23 (1978), 333-338.

[2] Abraham-Shrauner B \& Guo A, Hidden symmetries associated with the projective group of nonlinear first-order ordinary differential equations, Journal of Physics A: Mathematical and General, 25 (1992), 5597-5608.

[3] Abraham-Shrauner B \& Leach PGL, Hidden symmetries of nonlinear ordinary differential equations, in Exploiting Symmetry in Applied and Numerical Mathematics, Algower E, Georg K \& Miranda R, edd, Lectures in Applied Mathematics 29, American Mathematical Society, Providence, 1993, 1-10.

[4] Abraham-Shrauner B, Private communication, January 1994.

[5] Andriopoulos K \& Leach PGL, Autonomous self-similar ordinary differential equations and the Painlevé connection, Journal of Mathematical Analysis and Application 328 (2007), 625639 . 
[6] Andriopoulos K \& Leach PGL, Symmetry and similarity properties of second-order ordinary differential equation of Lie's Type III, Journal of Mathematical Analysis and Application $\mathbf{3 2 8}$ (2007), 860-875.

[7] Berkovich LM, Transformation of variables as a method of finding exact invariant solutions of the Kolmogorov-Petrovskiu-Piskunov equation and related nonlinear heat equations, Soviet Mathematics Dokladi, 45 (1992), 146-151.

[8] Berkovich LM, Factorization as a method of finding exact invariant solutions of the Kolmogorov-Petrovskiu-Piskunov equation and the related Semenov and Zel'dovich equations, Soviet Mathematics Dokladi, 45 (1992), 162-167.

[9] Bluman GW \& Kumei S, Symmetries and Differential Equations, Springer-Verlag, New York, 1989.

[10] Broucke R, Notes on the central force $r^{n}$, Astrophysics and Space Science, 72 (1980), 33-53.

[11] Chandrasekar VK, Senthilvelan M \& Lakshmanan M, On the general solution for the modified Emden type equation $\ddot{x}+\alpha x \dot{x}+\beta x^{3}=0$ Journal of Physics A: Mathematical and Theoretical (2007) (to appear)

[12] Clarkson P, References for the Painlevé Equations, University of Exeter preprint, 1994, 1-33.

[13] Conte R, Partial integrability of damped, forced, anharmonic oscillators, preprint SPEC/1991/063 Centre d'Études de Saclay, 1991, 0-8.

[14] Conte R, Singularities of differential equations and integrability, in Introduction to Methods of Complex Analysis and Geometry for Classical Mechanics and Nonlinear Waves, D. Benest and C. Froeschlé, edd., Éditions Frontières, Gif-sur-Yvette, 1994, 49-143.

[15] Emden R, Gaskugeln, Anwendungen der mechanischen Warmen-theorie auf Kosmologie und meteorologische Probleme, Leipzig, Teubner, 1907.

[16] Ermakov V, Second order differential equations. Conditions of complete integrability, Universita Izvestia Kiev Series III, 9 (1880), 1-25 (translated by A. O. Harin).

[17] Euler Norbert, Transformation properties of $\ddot{x}+f_{1}(t) \dot{x}+f_{2}(t) x+f_{3}(t) x^{n}=0$, Journal of Nonlinear Mathematical Physics 4 (1997), 310-337.

[18] Euler N \& Leach PGL, First integrals and reduction of a class of nonlinear higher order ordinary differential equations, Journal of Mathematical Analysis and Applications, 287 (2003), $337-347$.

[19] Feix MR, Geronimi C, Cairó L, Leach PGL, Lemmer RL \& Bouquet SÉ, Right and left Painlevé series for ordinary differential equations invariant under time translation and rescaling, Journal of Physics A: Mathematical and General, 30 (1997), 7437-7461.

[20] Fowler RH, The form near infinity of real, continuous solutions of a certain differential equation of the second order, Quarterly Journal of Mathematics, 45 (1914), 289-350.

[21] Fowler RH, Some results of the form near infinity of real, continuous solutions of a certain type of second order differential equations, Proceedings of the London Mathematical Society, 13 (1914), 341-371.

[22] Fowler RH, The solution of Emden's and similar differential equations, Monthly Notices of the Royal Astronomical Society, 91 (1930), 63-91. 
[23] Fowler RH, Further studies of Emden's and similar differential equations, Quarterly Journal of Mathematics (Oxford), 2 (1931), 259-288.

[24] Gambier B, Sur les équations différentielles du second ordre et du premier degré dont l'intégrale générale est a points critiques fixes, Acta Mathematica, 33 (1909), 1-55.

[25] Géronimi C, Leach PGL \& Feix MR, Singularity analysis and a function unifying the Painlevé and $\Psi$ series, Journal of Nonlinear Mathematical Physics, 9 S-2 (2002), 36-48.

[26] Govinder KS, On the equivalence of "non-equivalent" algebraic realisations, preprint: Astrophysics and Cosmology Research Unit, University of KwaZulu-Natal, Durban 4001, South Africa.

[27] Govinder KS \& Leach PGL, A Lie and Painlevé assault on the Emden-Fowler equation of index two, in Proceedings of the International Workshop: From Ordinary Differential Equations to Deterministic Chaos, Brüning E, Maharaj M \& Ori R, edd, University of Durban-Westville Press, Durban, 1994, 99-108.

[28] Govinder KS, Leach PGL \& Maharaj SD, Integrability analysis of a conformal equation arising in general relativity, International Journal of Theoretical Physics 34 (1994), 625-639.

[29] Govinder KS \& Leach PGL, On the determination of nonlocal symmetries, Journal of Physics A: Mathematical and General, 28, (1995), 5349-5359.

[30] Govinder KS \& Leach PGL, An elementary demonstration of the existence of $s \ell(3, R)$ for all second order linear ordinary differential equations, SIAM Review, 40 (1998), 945-946.

[31] Guo A \& Abraham-Shrauner B, Hidden symmetries of energy-conserving differential equations, IMA Journal of Applied Mathematics, 51 (1993), 147-153.

[32] Havas P, Shear-free spherically symmetric perfect fluid solutions with conformal symmetry, General Relativity and Gravitation, 24 (1992), 599-615.

[33] Head AK, LIE, a PC program for Lie analysis of differential equations, Computer Physics Communications, 77 (1993), 241-248.

[34] Hua DD, Cairó L, Feix MR, Govinder KS \& Leach PGL, Connection between the existence of first integrals and the Painlevé property in two-dimensional Lotka-Volterra and Quadratic Systems, Proceedings of the Royal Society of London, 452 (1996), 859-880.

[35] Ince EL, Ordinary Differential Equations, Longmans, Green and Co Ltd, London, 1927.

[36] Kara AH \& Mahomed FM, Equivalent Lagrangians and the solution of some classes of nonlinear equations $\ddot{q}+p(t) \dot{q}+r(t) q=\mu \dot{q} 2 q^{-1}+f(t) q^{n}$, International Journal of Nonlinear Mechanics, 27 (1992), 919-927.

[37] Kara AH \& Mahomed FM, A note on the solutions of the Emden-Fowler equation, International Journal of Nonlinear Mechanics, 28 (1993), 379-384.

[38] Kowalevski S, Sur les problème de la rotation d'un corps solide autour d'un point fixe, Acta Mathematica, 12 (1889), 177-232.

[39] Kowalevski S, Sur une propriété du système d'équations différentielles qui définit la rotation d'un corps solide autour d'un point fixe, Acta Mathematica, 14 (1889), 81-93. 
[40] Kummer EE, De generali quadam æquatione differentiali tertii ordinis, Journal für die Reine und Angewandte Mathematik, 100 (1887), 1-9 (reprinted from the Programm des evangelischen Königl und Stadtgymnasiums in Liegnitz for 1834).

[41] Lane IJ Homer, On the theoretical temperature of the sun under the hypothesis of a gaseous mass maintaining its volume by its internal heat and depending on the laws of gases known to terrestial experiment, American Journal of Science and Arts, 4 (1869-1870), 57-74.

[42] Leach PGL, First integrals for the modified Emden equation $\ddot{q}+\alpha(t) \dot{q}+q^{n}=0$, Journal of Mathematical Physics, 26 (1985), 2510-2514.

[43] Leach PGL, Maartens R \& Maharaj SD, Self-similar solutions of the generalized EmdenFowler equation, International Journal of Nonlinear Mechanics, 27 (1992), 575-582.

[44] Leach PGL \& Govinder KS, Hidden symmetries and integration of the generalized EmdenFowler equation of index two Proceedings 14th IMACS World Congress on Computational and Applied Mathematics, Ames WF ed , Georgia Institute of Technology, Atlanta, (1994), $300-303$.

[45] Lemmer RL \& Leach PGL, The Painlevé test, hidden symmetries and the equation $y^{\prime \prime}+y y^{\prime}+$ $k y 3=0$, Journal of Physics A: Mathematical and General, 26 (1993), 5017-5024.

[46] Lemmer RL \& Leach PGL, The Lie analysis and solutions for a class of second-order nonlinear differential equations, International Journal of Nonlinear Mechanics, 29 (1994), 177-185.

[47] Lie S, Vorlesungen über Continuierliche Gruppen mit Geometrischen und Anderen Anwendungen (Bearbeitet und Herausgegeben von G Scheffers), Chelsea, New York, 1971.

[48] Liouville J, Sur le développement des functions ou parties de fonctions en séries dont les divers termes sont assujettis à satisfaire à une même équation différentielle du second ordre contenant un paramètre variable, Journal de Mathématiques pures et appliquées, II (1837), $16-35$.

[49] Maharaj A \& Leach PGL, Properties of the dominant behaviour of quadratic systems, Journal of Nonlinear Mathematical Physics, 13 (2006), 129-144.

[50] Mahomed FM \& Leach PGL, Symmetry Lie algebras of $n$th order ordinary differential equations, Journal of Mathematical Analysis and Applications, bf 151 (1990), 80-107.

[51] Maharaj SD, Leach PGL \& Maartens R, Shear-free spherically symmetric solutions with conformal symmetry, General Relativity and Gravitation, 23 (1991), 261-267.

[52] Mellin CM, Mahomed FM \& Leach PGL, Solution of generalized Emden-Fowler equations with two symmetries, International Journal of Nonlinear Mechanics, 29 (1994), 529-538.

[53] Moyo S \& Leach PGL, Reduction Properties of Ordinary Differential Equations of Maximal Symmetry, (2006).

[54] Olver PJ, Applications of Lie groups to differential equations, Springer-Verlag, New York, 1993.

[55] Painlevé $\mathrm{P}$, Sur les équations différentielles du second ordre et d'ordre supérieur dont l'intégrale générale est uniforme, Acta Mathematica, 25 (1900), 1-85. 
[56] Painlevé P, Mémoire sur les équations différentielles dont l'intégrale générale est uniforme, Bulletin of the Mathematical Society of France, 28 (1900), 201-261. (This is just one example of a number of papers devoted to the analysis. See [12] for a detailed listing of references on the Painlevé property.)

[57] Pinney E, The nonlinear differential equation $y^{\prime \prime}(x)+p(x) y+c y^{-3}=0$, Proceedings of the American Mathematical Society, 1 (1950), 681.

[58] Ramani A, Grammaticos B \& Bountis T, The Painlevé property and singularity analysis of integrable and nonintegrable systems, Physics Reports, 180 (1989), 159-245.

[59] Srivastava DC, Exact solutions for shear-free motion of spherically symmetric perfect fluid distributions in general relativity, Classical and Quantum Gravity 4 (1987), 1093-1117.

[60] Whittaker ET, A Treatise on the Analytical Dynamics of Particles and Rigid Bodies, Dover, New York, 1944.

[61] Wong JSW, On the generalized Emden-Fowler equation, SIAM Review, 17 (1975), 339-360. 